\title{
TIME TO ARRIVAL ESTIMATES, (PEDESTRIAN) GAP ACCEPTANCE AND THE SIZE ARRIVAL EFFECT
}

\author{
Tibor Petzoldt ${ }^{1}$, Quyen Hoang-Sen Ngoc $^{2} \&$ Katja Bogda ${ }^{2}$ \\ ${ }^{1}$ TU Dresden, Germany \\ ${ }^{2}$ TU Chemnitz, Germany \\ Email: tibor.petzoldt@tu-dresden.de
}

\begin{abstract}
Summary: Various studies have found that road users' acceptance of gaps to cross in front of another vehicle is dependent on the approaching vehicle's size, with smaller accepted gaps in front of smaller vehicles. At the same time, the so called size arrival effect is well known from research on time to collision / time to arrival estimates, where larger objects / vehicles tend to be judged as arriving earlier than smaller objects / vehicles. However, so far there has been no attempt to connect these two approaches in a single experiment to investigate whether the size arrival effect that is prevalent in time to arrival estimates can explain the variations in gap acceptance. In this experiment, twenty-seven participants observed video clips of approaching virtual vehicles of varying size (truck, bus, van, two different cars and a motorcycle) from a pedestrian's perspective, and were either required to indicate a crossing decision, or to estimate time to arrival. While, overall, the effect of vehicle size was clearly visible for both crossing decision and time to arrival estimates, there was also a clear exception in form of the motorcycle, which went with larger accepted gaps than some of the larger vehicles. This exception might be explained by the participants' subjective rating of perceived threat, which was rather high for the motorcycle. As (with the exception of the motorcycle), vehicle size and perceived threat correlated substantially, it is unclear at this stage to what degree these two factors contribute to perceived time to arrival and crossing decisions.
\end{abstract}

\section{INTRODUCTION}

The assessment of "the time remaining before something reaches a person or particular place" (Tresilian, 1995) has been described as a competence that is central to a variety of activities in which a "collision" with an object is either pursued or must be avoided. In the context of road traffic, judging this time to arrival, or TTA, is obviously of vital importance. (In the literature, you also find the terms time to collision, time to contact, time to passage or arrival time, which all, more or less, describe the same concept. For reasons of consistency, the term time to arrival (or TTA) is used throughout this paper, as it best fits the experimental setup, and as it is broad enough to cover all the other terms. However, it has to be acknowledged that cited authors might have used different terminology.) While we are, overall, rather capable when it comes to the estimation of TTA, it is generally agreed that there are also a variety of factors that can have a systematic influence on such estimates (see Hecht \& Savelsbergh, 2004, for an overview).

One aspect that appears to be of specific practical relevance in road traffic is the influence of the approaching vehicle itself. Road users have to deal with a large variety of vehicles on a daily basis, vehicles that differ in design, performance, and, of course, size. It has repeatedly been found that the larger the approaching vehicle, the smaller the estimated TTA (e.g. Caird \& 
Hancock, 1994; Horswill, Helman, Ardiles, \& Wann, 2005). This so called size arrival effect had before been described by DeLucia (1991) for simple objects without any relation to the traffic context, and has been replicated repeatefly. Based on these findings, DeLucia (2013) suggested that the size arrival effect might play a role in road traffic crashes especially with smaller oncoming vehicles.

And indeed, vehicle size seems to impact also on road users' actual behaviour. Bottom and Ashworth (1978) observed motorists in real traffic accepting shorter gaps when confronted with smaller cars compared to larger ones. Keskinen, Ota and Katila (1998) observed a similar effect, with significantly shorter accepted gaps in front of motorcycles. Driving simulator studies showed the same results (e.g., Alexander, Barham, \& Black, 2002). For pedestrians' crossing decisions, only a few studies addressed the issue of vehicle size, and found that larger vehicles went with fewer crossings (Himanen \& Kulmala, 1988), or that critical gap size increased with vehicle length (Das, Manski, \& Manuszak, 2005).

However, in none of the studies on gap acceptance is the link between these findings and the size arrival effect in TTA estimates made explicitly. Instead, other concepts like the "expected cost of an accident", which "may depend on whether the oncoming vehicle is a rickshaw or a bus" (Das et al., 2005; p. 545) have been suggested as possible explanations. To our knowledge, the only study that has dealt with perceived TTA in the context of a gap acceptance study has been conducted by Petzoldt (2014), who found that the effect of vehicle approach speed on TTA estimates can be used to explain a similar effect of speed on pedestrians' gap acceptance behaviour. Based on this approach, aim of the experiment presented in this paper was to assess the relationship between TTA estimates and pedestrian crossing decisions, with specific focus on the size arrival effect. Based on previous findings, there should be a size arrival effect for both tasks, with larger vehicles estimated to arrive earlier than smaller ones, and smaller gaps accepted in front of smaller vehicles compared to larger ones. If the size arrival effect in TTA estimates was the main reason behind a similar effect in crossing decisions, the patterns of results should be complementary.

\section{METHOD}

\section{Participants}

Twenty-seven students from Technische Universität Chemnitz took part in this experiment. Twenty participants were female and seven male, with a mean age of 22.7 years $(\mathrm{SD}=3.6)$. All participants had normal or corrected-to-normal vision. They received course credits or monetary compensation for their participation.

\section{Material}

We created short video sequences, recorded from a pedestrian's point of view, of a virtual vehicle approaching at either 30 or $50 \mathrm{~km} / \mathrm{h}$ in an urban environment. Sequences were $2.4 \mathrm{~s}$ long, followed by a blank screen. TTA at the moment the screen was blanked ranged from $1.6 \mathrm{~s}$ to $7.2 \mathrm{~s}$ (in increments of $0.8 \mathrm{~s}$ ), resulting in eight different time gap sizes. Six different vehicles of various sizes and forms were used (see Figure 1 for an overview). To quantify the differences in 
size, we measured the visual angle of the vehicle front at $30 \mathrm{~km} / \mathrm{h}$ in a distance of $2 \mathrm{~s}$ (i.e., ca. $14.7 \mathrm{~m}$ ) from the observer (position as displayed in Figure 1).

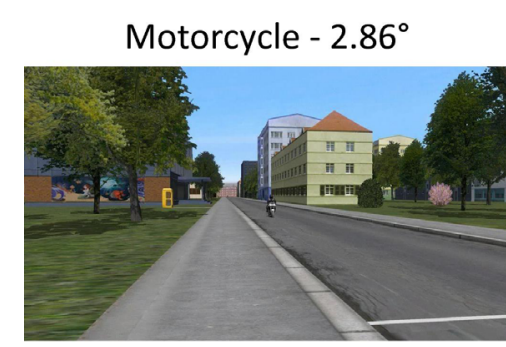

Van $-6.18^{\circ}$

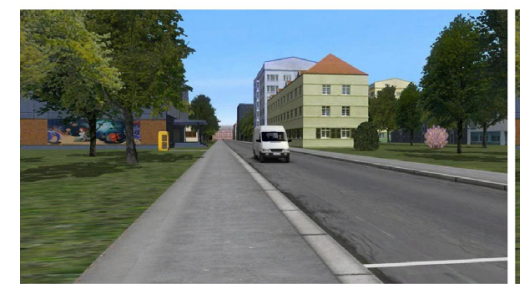

Car I $-4.12^{\circ}$

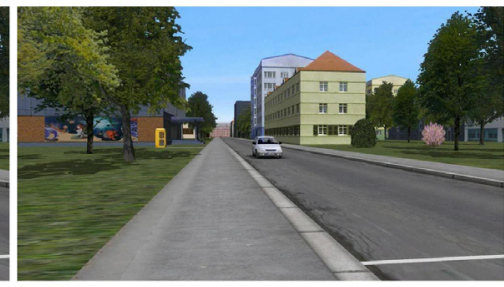

Bus $-8.81^{\circ}$

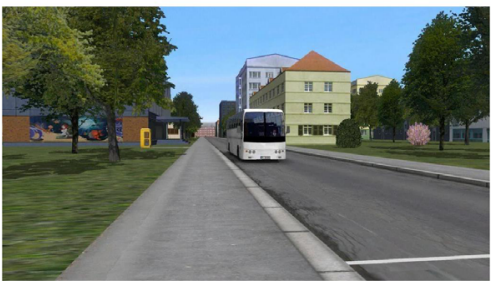

Car II $-4.58^{\circ}$

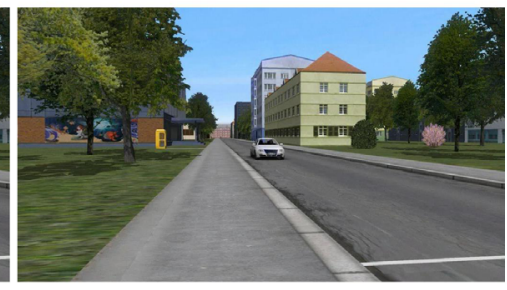

Truck $-9.60^{\circ}$

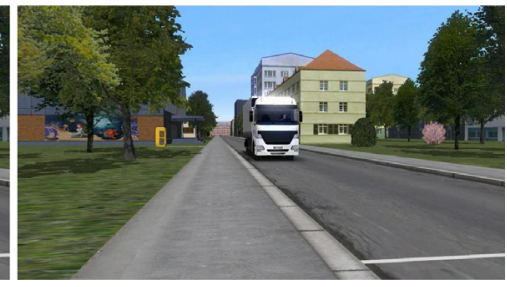

Figure 1. Screenshots of the vehicles used in the experiment. Distance from white reference line ca. $14.7 \mathrm{~m}$. Numbers indicate visual angle of vehicle in the screenshot as seen on the screen during the experiment.

Videos were presented on a 24" flat screen. Participants viewed exactly the same sequences in both a TTA estimation task and a crossing decision task. In the TTA estimation task, participants were asked to indicate the moment they felt the vehicle would have arrived at the white line (participants were told to consider the front of the vehicle as the reference for their decision). After a video sequence was presented (while the screen was blank), they were instructed to press the spacebar the moment they believed the vehicle had arrived. In the crossing decision task, participants were asked to state whether they would have crossed the street in front of the vehicle or not (at the position of the white line) at the moment the screen was blanked. They indicated their response by pressing one of two designated keys. The whole experiment was implemented using the E-Prime experimental suite.

In addition, we also obtained participants' subjective assessment of the vehicles' characteristics through a short questionnaire. Participants were required to rate the vehicles on a scale from 1 (no agreement) to 6 (full agreement) along a set of adjectives. The three items/adjectives that were of interest to us were "large" (which would reflect the participants" perception of the vehicles" physical dimensions), "dangerous" and "threatening" (both of which were expected to reflect to some degree the participants' perception of the potential risk of a crash with the respective vehicle). The adjectives also included a number of filler items (such as "rare" or "beautiful"), which were not intended for analysis.

\section{Procedure}

First, participants became acquainted with the nature of the video sequences. They were presented with some example screenshots and one video in order to familiarise them with the overall setting. Then, one of the two different tasks (crossing decision task or TTA estimation task) was explained, followed by three practice trials, before actual performance was measured 
on the first task. The same procedure (explanation, practice trials, measurement) was followed for the second of the two tasks. In each task, participants completed 96 experimental trials (6 vehicles x 2 speed levels x 8 TTA levels). After measurement, participants provided their ratings of the vehicles as well as demographic information via a short questionnaire. The whole experiment was completed in about $45 \mathrm{~min}$, with the order of tasks counter-balanced.

\section{Analysis}

To analyse the quality of participants' TTA estimates, we intended to collapse the data across the different TTA levels. This required a transformation of the raw estimates first. We calculated a TTA estimate ratio, which is the proportion of estimated TTA compared to actual TTA. A value above 1 indicates an overestimation of TTA, a value lower than 1 indicates an underestimation.

As a measure of participants' individual crossing decision behaviour, we calculated each participant's mean time gap accepted for both speeds and all six vehicles. To do so, participants' crossing decision patterns were used to create individual regression models to predict the probability that a certain gap is accepted for crossing in one of the experimental conditions by the respective participant. The TTA at which the probability of acceptance was $50 \%$ (the transition point of the logistic regression curve), was defined as the participant's mean time gap accepted (see Petzoldt, 2014). As this calculation requires that at least one gap (per factor level combination) is accepted and at least one declined, participants who either accepted all or none of the gaps of a certain factor level combination had to be excluded from the analysis. This was the case for six participants, which declined all gaps in either one of the truck or bus conditions.

In addition to traditional ANOVA, contrast analysis was used to test if the pattern of results corresponded with what would be expected if the size arrival effect was present (Abdi \& Williams, 2010; Rosenthal, Rosnow, \& Rubin, 2000). This method is especially useful when instead of simply testing for general differences in a dataset (like, e.g., an ANOVA would do), specific hypothesis can be quantified through so called contrast coefficients. In our case, the contrast coefficients describe how TTA estimates and mean accepted gap size were expected to vary dependent on vehicle type. As a basis for this expectation (our "prediction"), the visual angles of the different vehicles were used (see Figure 1). The data obtained in the TTA estimation and crossing decision tasks was then compared to the prediction. The fit of contrast coefficients and the dependent measures were calculated for each participant and the two different speed conditions as a correlation $r$, and one sample t-tests were computed (separately for the two different speed conditions) to investigate if the mean fit statistically differed from the null hypothesis (no fit). We always report the results of this test, as well as the average fit $\mathbf{r}_{\text {mean }}$.

\section{RESULTS}

In Figure 2 (left), mean TTA estimate ratios for the six different vehicles are displayed for both speed levels. As can be seen, there was a clear effect of speed on TTA estimates, with higher estimates being provided at $50 \mathrm{~km} / \mathrm{h}$. Also, there was a general trend for increasing estimates with decreasing vehicle size. The ANOVA confirmed this impression, with significant main effects of speed, $F(1,20)=88.50, p<.001, \eta^{2}=0.82$ as well as vehicle, $F(5,100)=11.54, p<$ $.001, \eta^{2}=0.37$. There also was a significant interaction between these two factors, $\mathrm{F}(5,100)=$ 
$2.33, \mathrm{p}=.048, \eta^{2}=0.10$. The additional contrast analyses (separately for the two speed levels) also provided significant results. For the $30 \mathrm{~km} / \mathrm{h}$ condition, the average correlation between participants' individual estimation patterns and the expected pattern based on visual angle was substantial, with $\mathrm{r}_{\text {mean }}=.61, \mathrm{t}(20)=11.01, \mathrm{p}<.001$. This was also the case, although with a smaller correlation, for the $50 \mathrm{~km} / \mathrm{h}$ condition, with $\mathrm{r}_{\text {mean }}=.40, \mathrm{t}(20)=3.81, \mathrm{p}=.001$.

Figure 2 (centre) shows mean accepted gap size in the crossing decision task as a function of vehicle and speed. Again, there was a strong effect of speed, as gaps accepted at $50 \mathrm{~km} / \mathrm{h}$ were smaller compared to the $30 \mathrm{~km} / \mathrm{h}$ condition, $\mathrm{F}(1,20)=63.38, \mathrm{p}<.001, \eta^{2}=0.76$. We also found a significant effect of vehicle type, $F(5,100)=7.72, p<.001, \eta^{2}=0.28$. There was no significant interaction between speed and vehicle type. The contrast analyses (separately for the two speed levels) again provided evidence for a considerable correlation between mean accepted gap size and actual vehicle size, with $\mathrm{r}_{\text {mean }}=.44, \mathrm{t}(20)=4.36, \mathrm{p}<.001$ for the $30 \mathrm{~km} / \mathrm{h}$ condition, and $\mathrm{r}_{\text {mean }}=.34, \mathrm{t}(20)=3.05, \mathrm{p}=.006$ for $50 \mathrm{~km} / \mathrm{h}$. The overall pattern was largely consistent with the results of the TTA estimation task. It has to be noted, however, that the motorcycle produced results that were at odds with the size arrival effect, with a mean accepted gap size larger than the gap size accepted for both cars at $50 \mathrm{~km} / \mathrm{h}$, and still larger than car II at $30 \mathrm{~km} / \mathrm{h}$.

To get a clearer indication of how the participants perceived the different vehicles subjectively, we analysed the three relevant items of the vehicle characteristics questionnaire. Figure 2 (right) shows the participants' ratings of vehicle size, danger and threat. The rating of size appears to reflect objective vehicle size quite accurately, as confirmed by the contrast analysis with $\mathbf{r}_{\text {mean }}=$ $.88, \mathrm{t}(20)=22.32, \mathrm{p}<.001$. In contrast, while the ratings for the four-wheeled vehicles again more or less reflect the vehicles' size, the subjective risk associated with the motorcycle is a clear outlier. Only the truck was perceived as more dangerous and threatening than the motorcycle. Contrast analyses for both items still show significant correlations to objective vehicle size, with $\mathrm{r}_{\text {mean }}=.30, \mathrm{t}(20)=3.32, \mathrm{p}=.003$ for danger, and $\mathrm{r}_{\text {mean }}=.54, \mathrm{t}(20)=7.37, \mathrm{p}<$ .001 for threat, but, compared to the size ratings, the effect is clearly reduced.
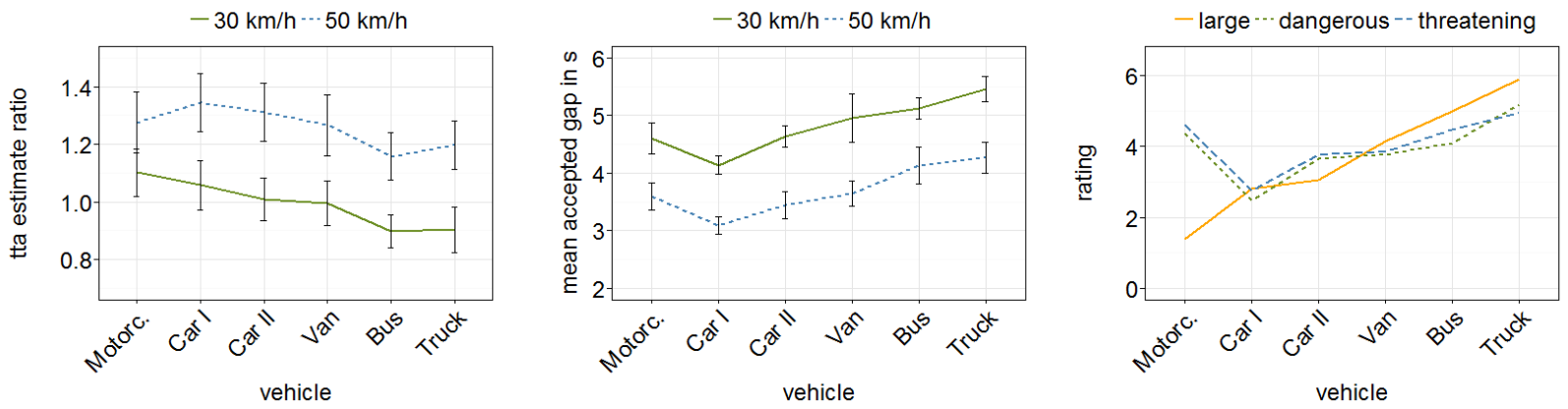

Figure 2. TTA estimate ratio (left), mean accepted gap size (centre) in s, and subjective ratings (right; 1 - no agreement, 6 - full agreement) for the six different vehicles and the two speed levels. Error bars represent standard error.

\section{DISCUSSION}

The results of the experiment seem to point towards a size arrival effect in both time to arrival estimation and crossing decision. Moreover, it appears that the effect was largely congruent for both measures, i.e. the effect of vehicle type on accepted gap size might, at least partially, be 
explained by differences in TTA estimates for the respective vehicles. However, while the overall effect of vehicle size was clearly present, it also appeared that there might be exceptions. The motorcycle, although by far the smallest vehicle used in the study, did not go with the smallest accepted gaps. Also, TTA estimation did not conform with the size arrival effect on the $50 \mathrm{~km} / \mathrm{h}$ speed level. It seems that mere object size might not be able to fully account for the differences between vehicles, especially with regard to the crossing decision.

As others have speculated (Das et al., 2005), one explanation for this exception might lie in the perceived risk or cost of a crash. Our participants' subjective assessment of threat and danger associated with the different vehicles showed that the motorcycle apparently was much more intimidating than most of the four-wheelers. The exact cause for this rating is, at this point, unclear. One might speculate that aspects such as typical speed or previous experience with the behaviour of drivers/riders of certain vehicles might play a role in this assessment, and consequently, also in the decision process. It is known that vehicle characteristics such as vehicle model can influence post-hoc attributions of vehicle speed (Davies, 2009; Davies \& Patel, 2005). Cherry and Andrade (2001) reported evidence that vehicle type and even colour influenced the speed estimated by an observer. It is reasonable to assume that the same characteristics also impact on perceived threat and crossing decisions. In fact, it has been suggested that perceived threat could also play a role in TTA estimates, albeit until now only for material unrelated to the traffic context (Brendel, DeLucia, Hecht, Stacy, \& Larsen, 2012; Brendel, Hecht, DeLucia, \& Gamer, 2014). There, it was concluded that arousal (induced by the stimulus whose approach is to be assessed) as elicited, e.g., by perceived threat, can have an impact on TTA judgments.

Unfortunately, as vehicle size often appears to be correlated with perceived threat at least partially, it seems that these two cannot easily be separated experimentally without additional manipulation. What is required is a setup that allows for variation of perceived threat independent of vehicle size. Ideally, such a setup would not rely on already acquired knowledge about certain types of vehicles as a source for variations in perceived threat. Instead, it should try to establish new links between vehicles and vehicle properties that might have an impact on perceived threat, to be able to study the effect of (perceived) threat in isolation.

It has to be acknowledged that the use of a video based approach was not ideal, as other perceptual cues that might be relevant in pedestrian crossing decisions were absent. The simple environment, with limited dynamic and without auditory information is certainly not representative of a real life crossing situation. Similarly, the reliance on young, healthy participants that are not suspected to have trouble crossing the road might be questioned. However, the fact that even these participants, who, given their perceptual capabilities, should be in the best position to assess the vehicles' approach regardless of other influencing factors (such as vehicle type / size), were susceptible to the effect of vehicle type / size in their responses indicates that the effect on other, less capable road users, could be even more severe. Nevertheless, it is obvious that our results would not necessarily directly translate into a more or less safe crossing behaviour, but can only indicate that certain characteristics of such crossing situations, e.g., the type of the approaching vehicle and the threat subjectively associated with that vehicle, might play a role in the overall decision process. 


\section{REFERENCES}

Abdi, H., \& Williams, L. J. (2010). Contrast analysis. In N. J. Salkind (Ed.), Encyclopedia of Research Design (pp. 243-251). Thousand Oaks, CA: Sage.

Alexander, J., Barham, P., \& Black, I. (2002). Factors influencing the probability of an incident at a junction: results from an interactive driving simulator. Accident Analysis and Prevention, 34(6), 779-792.

Bottom, C. G., \& Ashworth, R. (1978). Factors affecting the variability of driver gap-acceptance behaviour. Ergonomics, 21(9), 721-734.

Brendel, E., DeLucia, P. R., Hecht, H., Stacy, R. L., \& Larsen, J. T. (2012). Threatening pictures induce shortened time-to-contact estimates. Attention, Perception, \& Psychophysics, 74(5), 979-987.

Brendel, E., Hecht, H., DeLucia, P. R., \& Gamer, M. (2014). Emotional effects on time-tocontact judgments: arousal, threat, and fear of spiders modulate the effect of pictorial content. Experimental Brain Research, 232(7), 2337-2347.

Caird, J. K., \& Hancock, P. A. (1994). The perception of arrival time for different oncoming vehicles at an intersection. Ecological Psychology, 6(2), 83-109.

Cherry, T. L., \& Andrade, P. (2001). Bright cars and outsiders: evidence of asymmetric estimates in vehicular speeds. Journal of Applied Social Psychology, 31(12), 2538-2544.

Das, S., Manski, C. F., \& Manuszak, M. D. (2005). Walk or wait? An empirical analysis of street crossing decisions. Journal of Applied Econometrics, 20(4), 529-548.

Davies, G. M. (2009). Estimating the speed of vehicles: the influence of stereotypes. Psychology, Crime \& Law, 15(4), 293-312.

Davies, G. M., \& Patel, D. (2005). The influence of car and driver stereotypes on attributions of vehicle speed, position on the road and culpability in a road accident scenario. Legal and Criminological Psychology, 10(1), 45-62.

DeLucia, P. R. (1991). Pictorial and motion-based information for depth perception. Journal of Experimental Psychology: Human Perception and Performance, 17(3), 738-748.

DeLucia, P. R. (2013). Effects of size on collision perception and implications for perceptual theory and transportation safety. Current Directions in Psychological Science, 22(3), 199204.

Hecht, H., \& Savelsbergh, G. J. P. (2004). Time-to-contact. Elsevier.

Himanen, V., \& Kulmala, R. (1988). An application of logit models in analysing the behaviour of pedestrians and car drivers on pedestrian crossings. Accident Analysis \& Prevention, 20(3), 187-197.

Horswill, M. S., Helman, S., Ardiles, P., \& Wann, J. P. (2005). Motorcycle accident risk could be inflated by a time to arrival illusion. Optometry and Vision Science, 82(8), 740-746.

Keskinen, E., Ota, H., \& Katila, A. (1998). Older drivers fail in intersections: Speed discrepancies between older and younger male drivers. Accident Analysis \& Prevention, $30(3), 323-330$.

Petzoldt, T. (2014). On the relationship between pedestrian gap acceptance and time to arrival estimates. Accident Analysis and Prevention, 72, 127-133.

Rosenthal, R., Rosnow, R. L., \& Rubin, D. B. (2000). Contrasts and Effect Sizes in Behavioral Research: A Correlational Approach. Cambridge, UK: Cambridge University Press. 